\title{
SUPER QUASI-ADEQUATE SEMIGROUPS
}

\author{
R. J. WARNE
}

Let $S$ be a semigroup and let $L$ denote Green's relation on $S$, For $a, b \in S$, let $(a, b) \in L^{*}$ if and only if $(a, b) \in L$ in some cversemigroup of $S . R^{*}$ is defined dually and let $H^{*}=L^{*} \cap R^{*}$. From ([11] or [12]), $(a, b) \in L^{*}$ if and only if, for all $x, y \in S^{1}$ (S with an appended identity), $a x=a y$ if and only if $b x=b y$. So $L^{*}$ is a right congruence relation and $R^{*}$ is a left congruence relation. Fountain [9] terms a semigroup $S$ abundant if each $L^{*}$-class of $S$ and each $R^{*}$-class of $S$ contains an idempotent, and Fountain [9] terms $S$ superabundant if each $H^{*}$-class of $S$ contains an idempotent. If $S$ is a regular semigroup, $L^{*}=L$ and $R^{*}=R$. Hence, regular semigroups are abundant semigroups and unions of groups are superabundant semigroups.

In [9], Fountain gave superabundant analogues to the Rees Theorem and Clifford's well known theorem that a semigroup is a union of groups if and only if it is a semilattice of completely simple semigroups. In [7], El-Qallali terms an abundant semigroup $S$ to be $L^{*}$-unipotent if $E(S)$, the set of idempotents of $S$, form a subsemigroup and each $L^{*}$ class of $S$ contains precisely one idempotent. In [7], El-Qallali gives a structure theorem for super $L^{*}$-unipotent semigroups on which $H^{*}$ is a congruence ( $L^{*}$-unipotent bands of cancellative monoids [7]). A semigroup $S$ is termed $L$-unipotent if each $L$-class of $S$ contains precisely one idempotent (equivalently, $S$ is orthodox and each $J$-class of $E(S)$ is a right zero semigroup [20]). El-Qallali's theorem is a superabundant analogue to Bailes' structure theorem for $L$-unipotent union of groups on which $H$ is a congruence ( $L$-unipotent bands of groups) [1].

Let $S$ be an abundant semigroup. Fountain [8] terms $S$ an adequate semigroup if $E(S)$ is a semilattice. El-Qallali and Fountain [6] terms $S$ a quasi-adequate semigroup if $E(S)$ is a subsemigroup. If, furthermore, $L$ is a congruence relation on $E(S)$, we term $S$ a generalized $L^{*}$-unipotent semigroup. El-Qallali and Fountain [5], term a congruence $e$ on $S$ good if a $L^{*} b$ implies $a e L^{*} b e$ and $a R^{*} b$ implies $a e R^{*} b e$.

In section 1 , we give a structure theorem for super quasi adequate semigroups $S$ (Theorem 1.11). We first specialize the above mentioned results of Fountain to super quasi-adequate semigroups $S$. In particular, $S$ is a semilattice $Y$ of semigroups $\left(S_{y}\right.$ : $y \in Y$ ) where $S_{y}=T_{y} \times E\left(S_{y}\right)$ (algebraic direct product) where $T_{y}$ is a cancellative monoid and $E\left(S_{y}\right)$ is a rectangular band (Lemma 1.1). For $(g ; i, j),(h ; r, s) \in S$, define

Received January 13, 1989. 
$(g ; i, j) \delta(h ; r, s)$ if $(g ; i, j),(h ; r, s) \in S_{y}$, say, and $g=h$. Then, $\delta$ is the minimum adequate good congruence on $S$ (Proposition 1.3) and $S / \delta$ is a strong semilattice $Y$ of the $T_{y}$ (Lemma 1.4). Then, $S^{1}$ divides $V \circ(\widehat{S / \delta})^{1}$ where $V$ is an $L$-trivial and idempotent monoid, o is wreath product, ${ }^{-}$is the Rhodes expansion, $(\widehat{S / \delta})$ is a semilattice $Y$ of left cancellative semigroups $\left(X_{y}: y \in Y\right)$ with idempotents, and $E((\widehat{S / \delta}))$ is a semilattice $Y$ of right zero semigroups $\left(E\left(X_{y}\right): y \in Y\right.$ ) (Theorem 1.11). If $S$ is an orthodox union of groups, $\delta$ becomes the smallest inverse semigroup congruence on $S, T_{y}$ becomes a maximal subgroup of $S$, and $X_{y}=T_{y} \times E\left(X_{y}\right)$ (algebraic direct product) (see Lemma 1.12). Hence, Theorem 1.11 is a superabundant semigroup analogue to our structure theorem for orthodox unions of groups [26].

In section 2, we give a structure theorem for super generalized $L^{*}$-unipotent semigroups $S$ (Theorem 2.4). We first show that $\delta \cap L$ is the smallest $L^{*}$-unipotent good congruence on $S$ and $S / \delta \cap L$ is a semilattice $Y$ of the semigroups $\left(\left(T_{y} \times J_{y}\right): y \in Y\right)$ were $J_{y}$ is an $R$-class of $E\left(S_{y}\right)$ (Proposition 2.1). Then,

$$
\begin{aligned}
S & \leq W^{1} \circ(E(S) / L)^{1} \circ(S / \delta \cap L)^{1} \\
& (\leq \text { means "is embedded in") and } S / \delta \cap L \\
& \leq(S / \delta \cap L / e)^{1} \stackrel{\Omega}{\circ}(E(S) / L)^{1}
\end{aligned}
$$

where $W$ is a lower partial chain $Y$ of left zero subsemigroups of $E(S), e$ is the smallest adequate good congruence on $S / \delta \cap L, S / \delta \cap L / e$ is a strong semilattice $Y$ of the $T_{y}$, and $\circ$ is reverse wreath product (Theorem 2.4). An orthodox semigroup $S$ is termed generalized $L$-unipotent if $L$ is a congruence relation on $E(S)$. If $S$ is a generalized $L$-unipotent union of groups, $\delta \cap L$ becomes the smallest $L$-unipotent congruence on $S$. Hence, Theorem 2.4 is a superabundant analogue to our structure theorem for generalized $L$-unipotent unions of groups [24].

In section 3 , we show that if $S$ is a super $R^{*}$-unipotent semigroup, then $S \leq(E(S))^{1}$ o $(S / \delta)^{1}$ where $E(S)$ is a semilattice $Y$ of left zero semigroups (Theorem 3.1). Theorem 3.1 is a superabundant analogue to our structure theorem for $R$-unipotent unions of groups [24].

Abundant semigroup analogues to many theorems in regular semigroup theory have been given by Fountain ([8], [9]), El-Qallali and Fountain ([5], [6]), and El-Qallali [7].

We have studied the structure of generalized $L$-unipotent semigroups in ([21], [22], [23], [24]), $R$-unipotent semigroups have been studied extensively by many authors-most recently by Szendrei ([14], [15]).

A submonoid of a monoid $S$ is a subsemigroup of $S$ containing the identity of $S$.

A semigroup (monoid) $S$ is said to divide a semigroup (monoid) $T$ if there exists a homomorphism of a subsemigroup (submonoid) of $T$ onto $S$. We also say $T$ covers $S$ in this case and write $S<T$. If there exists an isomorphism of $S$ into $T$, we write $S \leq T . R, L, H, D$ and $J$ will denote Green's relations and $E(S)$ will denote the set of idempotents of a semigroup $S$. 
See [9] for the definition of $J^{*}$. If $S$ is a regular semigroup $J^{*}=J$.

We adopt the following notation and definitions from [24, p. 181-182]: $S^{1}$ ( $S$ with appended identity), $S^{\circ}$, wreath product "o" of semigroups, reverse wreath product " ${ }_{0}$ " of semigroups, type $A$ semigroup congruence (for example, inverse semigroup congruence), ae ( $a \in S$, a semigroup) ( $e$, a congruence on $S$, will also denote the natural homomorphism of $S$ onto $S / e)$, and unions of groups.

For other definitions not given in this paper, see [2] or [10]. We also adopt the notation of [2] unless otherwise specified.

A monoid $S$ is termed $L$-trivial and idempotent if each $L$-class of $S$ is a singleton and $S$ is a band.

\section{Section 1 - The Structure of Super Quasi-Adequate Semigroups}

In this section, we describe the minimum adequate good congruence $\delta$ on a super quasi-adequate semigroup (Proposition 1.3 and Lemma 1.4) and give a structure theorem for super, quasi-adequate semigroups (Theorem 1.11).

Let $S$ be a semigroup. For ${ }_{a} \in S, L_{a}^{*}$ or $L_{a}^{*}(S)$ (in case of ambiguity) will denote the $L^{*}$-class of $S$ containing $a$ (notation of [9]).

Let $S$ be a semigroup and $I$ and $J$ be sets and let $P: J \times I \longrightarrow S$ with $(j, i) P=p_{j i}$. Let $M(S, I, J, P)$ denote $S \times I \times J$ under the multiplication $\left(a ;_{i, j}\right)\left(b ;_{r, s}\right)=\left(a p_{j r} b ; ;_{i, s}\right)$. We term $M(S, I, J, P)$ a Rees Matrix semigroup over $S$ with entries in $P$.

The following lemma gives the "gross" structure of super quasi-adequate semigroups.

Lemma 1.1. A semigroup $S$ is super quasi-adequate if and only if $S$ is a semilattice $Y=S / J^{*}$ of semigroups $\left(S_{y}: y \in Y\right)$ where $S_{y}=T_{y} \times E\left(S_{y}\right)$ where $T_{y}$ is a cancellative monoid and $E\left(S_{y}\right)$ is a rectangular band, $L_{a}^{*}(S)=L_{a}^{*}\left(S_{y}\right)$ and $R_{a}^{*}(S)=R_{a}^{*}\left(S_{y}\right)$ for $y \in Y$ and $a \in S_{y}$ and $E(S)$ is a semilattice $Y$ of rectangular bands $\left(E\left(S_{y}\right):_{y} \in Y\right)$.

Proof. Utilizing [9, Theorem 6.8 and its proof and Corollary 5.2], we obtain the above theorem (except the statement about $E(S)$ ) with $S_{y}=M\left(T_{y}, I_{y}, J_{y}, P_{y}\right.$ ), a Rees matrix semigroup over a cancellative monoid $T_{y}$ where the entries of $P_{y}$ are units $U$ of $T_{y}$. As is easily shown, [2, Lemma 3.6] is valid for the above matrix semigroups if we require the mappings to have range $U$. Using this Lemma, we may "normalize" $P_{y}$ such that all the elements in a given row and a given column are the identity e of $T_{y}$. Then, using the assumption that $E(S)$ is a subsemigroup, we may show $p_{j i}=e$ for all $j \in J_{y}$ and $i \in I_{y}$. Hence, $M\left(T_{y}, I_{y}, J_{y}, P_{y}\right)=T_{y} \times E\left(S_{y}\right)$ where $E\left(S_{y}\right)$ is a rectangular band.

To show $\delta$ is a congruence relation (Proposition 1.3), we will need the following lemma.

Lemma 1.2. Let $S_{y}=T_{y} \times E_{y}$ and $S_{x}=T_{x} \times I_{x} \times J_{x}$ where $T_{y}$ and $T_{x}$ are cancellative monoids, $E_{y}$ is a rectangular band, $I_{x}$ is a left zero semigroup, and $J_{x}$ is a 
right zero semigroup. Assume these exists

(a) a left representation ${ }_{A} \longrightarrow \lambda_{A}$ of $S_{y}$ by transformations of $I_{x}$

(b) a right representation $A \longrightarrow e_{A}$ of $S_{y}$ by transformations of $J_{x}$

(c) a homomorphism $\phi$ of $T_{y}$ into $T_{x}$,

Define a binary operation on $S_{y} \cup S_{x}$ extending the given ones on $S_{y}$ and $S_{x}$ by defining products of $A=(a, e) \in S_{y}$ and $(b ; i, j) \in S_{x}$ as follows:

$$
\begin{aligned}
& (a, e)(b ; i, j)=\left(a \phi b ; \lambda_{A} i, j\right) \\
& (b ; i, j)(a, e)=\left(b(a \phi) ; i, j e_{A}\right) .
\end{aligned}
$$

Then, $S_{y} \cup S_{x}$ becomes a semigroup with $S_{x}$ an ideal.

Conversely every possible binary associative operation on $S_{y} \cup S_{x}$ extending the given ones on $S_{y}$ and $S_{x}$, and such that $S_{x}$ is an ideal, can be constructed in the above manner.

Proof. Lemma 1.2 has been established by Clifford [ 3, Lemma 2.5] in the case $T_{y}$ and $T_{x}$ are groups. Clifford's proof is easily seen to be valid when $T_{y}$ and $T_{x}$ are just cancellative monoids.

Proposition 1.3. Let $S$ be a super quasi-adequate semigroup. Then, $\delta$ is the minimum adequate good congruence on $S$.

Proof. We first show that $\delta$ is a congruence relation on $S$. Let $\bar{\delta}$ denote the smallest congruence on $S$ containing $\delta$. Suppose a $\bar{\delta} b$. Then, there exists $a=a_{1}, a_{2}, \ldots, a_{n}=b \in$ $S$ such that $a_{i}=x_{i} u_{i} y_{i}, a_{i+1}=x_{i} v_{i} y_{i}$ where $x_{i}, y_{i} \in S^{1}$ and $\left(u_{i}, v_{i}\right) \in \delta$ for $1 \leq i \leq n-1$. Let $x_{i}=(w ; i, j)_{\alpha} \in S_{\alpha}, y_{i}=(h ; r, s)_{\beta} \in S_{\beta}, u_{i}=(g ; m, n)_{\gamma}$, and $v_{i}=(g ; c, d)_{\gamma}$. Hence, $a_{i}=(A ; p, q)_{\alpha \beta \gamma} \in S_{\alpha \beta \gamma}$ and $a_{i+1}=(B ; k, l)_{\alpha \beta \gamma} \in S_{\alpha \beta \gamma}$ say. Let $\theta=_{\alpha \beta \gamma}$. Thus,

$$
\begin{aligned}
& (A ; p, q)_{\theta}=(w ; i, j)_{\alpha}(g ; m, n)_{\gamma}(h ; r, s)_{\beta} \\
& (B ; k, l)_{\theta}=(w ; i, j)_{\alpha}(g ; c, d)_{\gamma}(h ; r, s)_{\beta}
\end{aligned}
$$

Multiply both of the above equations on the left and right by $(e ; p, q)_{\theta}$ where $e$ is the identity of $T_{\theta}$.

Hence,

$$
\begin{aligned}
& (A ; p, q)_{\theta}=(\bar{W} ; \bar{i}, \bar{j})_{\theta}(g ; m, n)_{\gamma}(\bar{h} ; \bar{r}, \bar{s})_{\theta} \\
& (B ; p, q)_{\theta}=(\bar{W} ; \bar{i}, \bar{j})_{\theta}(g ; c, d)_{\gamma}(\bar{h} ; \bar{r}, \bar{s})_{\theta}
\end{aligned}
$$

say,

Using Lemma 1.2

$$
(A ; p, q)_{\theta}=\left(\bar{W}\left(g \omega_{\gamma, \theta}\right) ; \bar{i}, \bar{j} e_{(g, m, n) \gamma}\right)_{\theta}(\bar{h} ; r, s)_{\theta}=\left(\bar{W}\left(g \omega_{\gamma, \theta}\right) \bar{h} ; \bar{i}, s\right)_{\theta} .
$$

where $\omega_{\gamma, \theta}$ is the homomorphism of $T_{\gamma}$ into $T_{\theta}$ given by Lemma 1.2 and $(B ; p, q)_{\theta}=$ $\left.\overline{(W}\left(g \omega_{\gamma, \theta}\right) ; \bar{i}, \bar{j} e_{(g, c, d) \gamma}\right)_{\theta}(\bar{h} ; r, s)_{\theta}=\left(\bar{W}\left(g \omega_{\gamma, \theta}\right) \bar{h} ; \bar{i}, s\right)_{\theta}$. Hence, $A=B$. Thus $a_{i} \delta a_{i+1}$ for $1 \leq i \leq n-1$. Hence, $a \delta b$. Thus, $\bar{\delta}=\delta$, and, hence, $\delta$ is a congruence on $S$. 
Let $a \in S$ and let $a^{+}, a^{*} \in E(S)$ such that $a^{+} R^{*} a$ and $a^{*} L^{*} a$. Using [9, Corollary 6.2 and Proposition 6.5] and Lemma 1.1, $a^{+}, a^{*}, a \in S_{y}$, say. Hence, using [6, Corollary 2.4 and Proposition 2.6], $\delta$ is the minimum adequate good congruence on $S$.

Lemma 1.4. Let $S$ be a super quasi-adequate semigroup. Then, $S / \delta$ is a strong semilattice $Y$ of cancellative monoids $\left(T_{y} ; y \in Y\right)$.

Proof. Let $(\overline{g ; i, j})$ denote the $\delta$-class of $S$ containing $(g ; i, j)$. Since $(\overline{g ; i, j}) \tau=g$ defines a 1-1 map of $S / \delta$ onto $T=U\left(T_{y}:{ }_{y} \in Y\right), T$ becomes a groupoid under the multiplication $a b=\left(a, \tau^{-1} b \tau^{-1}\right) \tau$ and $\tau$ defines an isomorphism of $S / \delta$ onto $T$. If $g, h \in$ $T_{y}, g h=((\overline{g ; i, j})(\overline{h ; k, e})) \tau=(\overline{g h ; i, e}) \tau=g h$ (the last product is multiplication in $\left.T_{y}\right)$. Hence, $T$ is a semilattice $Y$ of cancellative monoids $\left(T_{y}: y \in Y\right)$. For $a \in T_{x}$ and $x \geq_{y}$, define a $\varsigma_{x, y}=a e_{y}$ where $e_{y}$ is the identity of $T_{y}$. It is routine to verify that $\varsigma_{x, y}$ is a homomorphism of $T_{x}$ into $T_{y}, \varsigma_{y, y}$ is the identity map on $T_{y}$, and, for $a \in T_{y}, b \in T_{x}, a b=a \varsigma_{y, y x} b \varsigma_{x, y x}$. Using the fact that the idempotents of $T$ commute by Proposition 1.3, its easily seen that $\varsigma_{y, x} \varsigma_{x, w}=\varsigma_{y, w}$ for $y \geq_{x} \geq_{w}$. Hence, $T$ is a strong semilattice $\varsigma_{(}\left(Y ; T_{y} ; c_{y, x}\right)$ of cancellative monoids (notation of [10]). We identify $S / \delta$ and T.

We next describe the Rhodes expansion $\widehat{S}$ of an arbitrary semigroup $S$ (see [17] and [13]). The Rhodes expansion and certain of its properties will be crucial in developing our structure theory of super quasi-adequate semigroups. If $a, b \in S, a \leq b$ means $a \cup S a \leq b \cup S b$ and $a<b$ means $a \leq b$ but $a \mathbb{L} b$. Let $S_{+}=\left\{\left(s_{n}, \ldots, s_{1}\right): s_{i} \in S\right.$ for $1 \leq i \leq n$ and $\left.s_{1} \leq s_{2} \leq \ldots \leq s_{n}\right\}$. If $x=\left(s_{n}, \ldots, s_{1}\right), y=\left(t_{m}, \ldots, t_{1}\right)$ define $x y=\left(s_{n} t_{m}, \ldots, s_{1} t_{m}, t_{m}, \ldots, t_{1}\right)$. Then, $S_{+}$is a semigroup under this multiplication. If $a=\left(s_{n}, \ldots, s_{1}\right) \in S_{+}$and $s_{k+1} L s_{k}$ for some $1 \leq k \leq n-1$ delete $s_{k}$ to obtain $a_{1} \in S_{+}$and denote the deletion by $a \longrightarrow a_{1}$. Perform $a \longrightarrow a_{1} \longrightarrow \ldots \longrightarrow a_{k}$ where $a_{k}=\left(s_{n}, s_{n 1}, \ldots, s_{n r}\right)$ with $s_{n}<s_{n 1}<\ldots<s_{n r}$ (such an $a_{k}$ is termed an irreducible element of $S_{+}$). Write $a_{k}=$ red $a$ and $a \sim b$ if red $a=$ red $b$. The equivalence relation $\sim$ is a congruence relation on $S_{+}$. I et $\widehat{S}=S_{+} / \sim . \widehat{S}$ is termed the Rhodes expansion of $S$ after its inventor John Rhodes. $\widehat{S}$ will be treated as the set of irreducible elements of $S_{+}$under the multiplication $a b=\operatorname{red}(a b)$.

Lemma 1.5. Let $S$ be a super quasi-adequate semigroup. Then, $\widehat{S}$ is a semilatice $Y$ of subsemigroups $\left(F_{y}:{ }_{y} \in Y\right)$ where $F_{y}=\left\{\left(a_{n}, a_{n-1}, \ldots, a_{1}\right): a_{n} \in S_{y}, a_{j} \in S\right\}$ and $\mathbb{E}(\widehat{S})$ is the semilattice $Y$ of rectangular bands

$$
E\left(F_{y}\right)=\left\{\left(\left(e_{y} ; i, j\right), a_{n-1}, \ldots, a_{1}\right):\left(e_{y} ; i, j\right) \in E\left(S_{y}\right), a_{j} \in S\right\} .
$$

$U=(\widehat{S / \delta})$ is a semilattice $Y$ of left cancellative semigroups with idempotent $\left(X_{y}:_{y} \in\right.$ $Y)$ where $X_{y}=\left\{\left(a_{n}, a_{n-1}, \ldots, a_{1}\right): a_{n} \in T_{y}, a_{j} \in S / \delta\right\} . E(U)$ is a semilattice $Y$ of right zero semigroups $\left(E\left(X_{y}\right):_{y} \in Y\right)$ where $E\left(X_{y}\right)=\left\{\left(e_{y}, a_{n-1}, \ldots, a_{1}\right): e_{y}\right.$, the identity of $\left.T_{y}, a_{j} \in S / \delta\right\}$. For $\left(a_{n}, a_{n-1}, \ldots, a_{1}\right) \in \widehat{S}$, let $\left(a_{n}, a_{n-1}, \ldots, a_{1}\right) \hat{\delta}=$ $\operatorname{red}\left(a_{n} \delta, a_{n-1} \delta, \ldots, a_{1} \delta\right)$. Then, $\widehat{\delta}$ defines a homomorphism of $\widehat{S}$ onto $(\widehat{S / \delta})$. 
Proof. To establish the second sentence of the lemma, utilize Lemma 1.1 and [16, Lemma 6.7] (see also [17, Lemma 11.4] and [24, Theorem 3.1(f)]). Utilizing Lemma 1.4 and [24, Theorem 3.1(f)], it is easily checked that $U$ is a semilattice $Y$ of the semigroups $\left(X_{y}{ }_{y} \in Y\right)$ and that the fourth sentence of the lemma is valid. We next show $X_{y}$ is left cancellative for $y \in Y$. Let $\left(x_{r}, x_{r-1}, \ldots, x_{1}\right),\left(a_{n}, a_{n-1}, \ldots, a_{1}\right)$, and $\left(b_{s}, b_{s-1}, \ldots, b_{1}\right)$ be elements of $X_{y}$ and suppose that $\left(x_{r}, x_{r-1}, \ldots, x_{1}\right) \cdot\left(a_{n}, a_{n-1}, \ldots, a_{1}\right)=\left(x_{r}, x_{r-1}, \ldots\right.$, $\left.x_{1}\right) \cdot\left(b_{s}, b_{s-1}, \ldots, b_{1}\right)$. Hence, $\operatorname{red}\left(x_{r} a_{n}, x_{r-1} a_{n}, \ldots, x_{1} a_{n}, a_{n}, a_{n-1}, \ldots, a_{1}\right)=\operatorname{red}\left(x_{r} b_{s}\right.$, $\left.x_{r-1} b_{s}, \ldots, x_{1} b_{s}, b_{s}, b_{s-1}, \ldots, b_{1}\right)$. Thus, $x_{r} a_{n}=x_{r} b_{s}$. Hence, since $T_{y}$ is a cancellative semigroup, $a_{n}=b_{s}$. Thus, $n=s$ and $a_{i}=b_{i}$ for $1 \leq i \leq n$. The last sentence of the lemma is a consequence of [16, Proposition 6.6] (see also [17] and [24, Theorem 3.11(b)]).

In the remainder of this section, $S$ will denote a super quasi-adequate semigroup.

If $A$ is a semigroup and $a=\left(a_{n}, \ldots, a_{1}\right) \in \widehat{A}$, let $|a|=n$. We term $|a|$ the length of a.

\section{Lemma 1.6. If $z \in \widehat{S},|z|=|z \widehat{\delta}|$}

Proof. Let $z=\left(a_{n}, a_{n-1}, \ldots, a_{1}\right)$. Suppose $a_{k+1} \delta L a_{k} \delta$ for some $1 \leq k \leq n-1$. Using Lemma 1.1, let $a_{k+1}=\left(g_{k+1} ; i_{k+1}, j_{k+1}\right) \in S_{y}$, say, and $a_{k}=\left(g_{k} ; \bar{i}_{k}, j_{k}\right) \in$ $S_{x}$, say. Thus, $a_{k+1} \delta=g_{k+1} \in T_{y}$ and $a_{k} \delta=g_{k} \in T_{x}$, and, hence, $g_{k+1} L g_{k}$ (in $S / \delta$ ). Using Lemma 1.4 , it easily seen that $y=z$ and $g_{k+1}=\mu g_{k}$ where $\mu$ is a unit of $T_{y}$. Since $a_{k+1}<a_{k}, a_{k+1}=s a_{k}$ for some $s \in S$. We may take $s=$ $\left(s^{\prime} ; m, n\right) \in S_{y}$. Hence, $\left(g_{; k+1} i_{k+1}, j_{k+1}\right)=\left(s^{\prime} ; m, n\right)\left(g_{k} ; i_{k}, j_{k}\right)$. So, $j_{k+1}=j_{k}$. Thus, $\left(g_{k} ; i_{k}, j_{k}\right)=\left(\mu^{-1} ; i_{k}, j_{k}\right) .\left(g_{k+1} ; i_{k+1}, j_{k+1}\right)$. Hence, $a_{k+1} L a_{k}$, a contradiction. Thus, $\operatorname{red}\left(a_{n} \delta, a_{n-1} \delta, \ldots, a_{1} \delta\right)=\left(a_{n} \delta, a_{n-1} \delta, \ldots, a_{1} \delta\right)$ and $|z|=|z \delta|$.

For ${ }_{t} \in U=(\widehat{S / \delta})$, let $U_{t}=\left\{x \in U:_{t} x={ }_{t}\right\}$

Lemma 1.7. For $t \in U, U_{t} \widehat{\delta}^{-1} \leq E(\widehat{S})$. If $f_{t} \in X_{y}, U_{t} \widehat{\delta}^{-1} \leq U\left(E\left(F_{x}\right):_{x} \geq_{y}\right)$.

Proof. Let $s \in U_{t} \widehat{\delta}^{-1}$. Hence, $s \widehat{\delta} \in U_{t}$. Using an important theorem of Rhodes [13, Theorem A.1V.1], $(s \hat{\delta})^{|t|+1}=(s \widehat{\delta})^{|t|}$. Let $s=\left(s_{n}, s_{n-1}, \ldots, s_{1}\right)$. Then, $s \widehat{\delta}=$ $\left(s_{n} \delta, s_{n-1} \delta, \ldots, s_{1} \delta\right)$. If $s_{n}=(g ; i, j) \in S_{y}, s_{n} \delta=g \in T_{y}$. Thus, $p r_{1}(s \hat{\delta})^{|t|+1}=g^{|t|+1}$ and $p r_{1}(s \hat{\delta})^{|t|}=g^{|t|}$. Let $e$ denote the identity of $T_{y}$. Thus, since $T_{y}$ is a cancellative monoid, $g^{|t|} e=g^{|t|} g$ implies $e=g$. Hence, $s_{n} \in E(S)$. Thus, using [24, Theorem 3.1(f)], $s \in E(\widehat{S})$. Hence $U_{t} \widehat{\delta}^{-1} \leq E(\widehat{S})$. The last sentence of the lemma is a consequence of the definitions of $U_{t}$ and $\delta$, Lemma 1.5 , and the first sentence of the lemma.

If we replace " $e$ " by " $\delta$ ", " $X_{y}$ " by " $F_{y}$ ", " $G_{y}$ " by " $T_{y}$ ", and " $U_{y}$ " by " $X_{y}$ " in [26, Lemma 5, Lemma 7, Lemma 8, Lemma 9, Lemma 11] (if $U_{t} \widehat{\delta}^{-1} \neq \phi$ and the last sentence is omitted), Lemma 12, Lemma 13, the first two sentences of Lemma 15, Lemma 16, Lemma 17, and Lemma 18 (with "and $\cdots Y^{x}$ " omitted)], these lemmas are valid for quasi-adequate semigroups $S$. The proofs of these modified lemmas are the same as the proofs of the original lemmas in [26] except that we replace Lemma 1 of [26] by Lemma 1.1, 1.4, and 1.5 and Proposition 1.3; Lemma 2 of [26] by Lemma 1.6; and Lemma 6 of 
[26] by Lemma 1.7 in the proofs of the original lemmas. Using Lemmas 1.1, 1.4 and 1.5, Proposition 1.3, Lemma 1.6, [26, Lemma 3], Lemma 1.7, and the modified Lemmas, we obtain

Lemma 1.8. If $U_{t} \widehat{\delta}^{-1} \neq \phi$, then $U_{t} \widehat{\delta}^{-1}$ is a chain $\widetilde{P}_{|t|}$ of rectangular bands $\left(W_{j}: j \in\right.$ $\left.\tilde{P}_{|t|}\right)$ where $\tilde{P}_{|t|}$ is a sub-chain of $P_{|t|}=\{1,2, \cdots,|i|\}$ under the reverse of the usual order. Furthermore, every element of $W_{j}$ has length ${ }_{j}$.

Let $t \in X_{y}$ and suppose that $|t|=_{k}$. If $x, y \in U_{t} \widehat{\delta}^{-1}$, define $x \sigma^{\prime} y$ if and only if $a æ=a y$ for all $a \in W_{k}$ where $k$ is thé least element of $\tilde{P}_{k}$.

If we make the usual modifications and furthermore replace " $\sigma$ " by " $\sigma$ ", [26, Lemma 21 and Lemma 23] are valid for super quasi-adequate semigroups $S$. The proofs also remain valid of we replace " $\sigma$ " by " $\sigma$ ", " $e$ " by " $\delta$ ", $k$ by $\bar{k}$, and Lemma 7 by modified Lemma 7 if we note that $e_{j} L g_{j}$ (notation of [26, Lemma 23]) by virtue of the modified Lemma 5.

Lemma 1.9. If $U_{t} \widehat{\delta}^{-1} \neq \phi, L$ is a congruence relation on $U_{t} \widehat{\delta}^{-1}$. Hence, $U_{t} \delta^{-1} / L$ is a chain $\tilde{P}_{|t|}$ of right zero semigroups $\left(W_{j} / L:_{j} \in \widetilde{P}_{|t|}\right)$.

Proof. Replace " $\delta$ " for " $e$ ", Lemmas 21 and 23 by their modifications, and Lemma 1.8 for Lemma 20 in the proof of [26, Lemma 24].

Let $r$ be a homomorphism of a monoid $S$ onto a monoid $T$, we define a category $R_{r}$ as follows: obj $\mathbb{R}_{r}=T$. For $t_{1}, t_{2} \in T, R_{r}\left(t_{1}, t_{2}\right)=\left\{\left(t_{1}, s, t_{2}\right): s \in S\right.$ and $t_{2}=$ $\left.t_{1}\left(s_{r}\right)\right\}$. For $\left(t_{1}, s_{1}, t_{2}\right) \in R_{r}\left(t_{1}, t_{2}\right)$ and $\left(t_{2}, s_{2}, t_{3}\right) \in R_{r}\left(t_{2}, t_{3}\right)$, we define the composition $\left(t_{1}, s_{1}, t_{2}\right)\left(t_{2}, s_{2}, t_{3}\right)=\left(t_{1}, s_{1} s_{2}, t_{3}\right)$. It is easily checked that $\left(t_{1}, s_{1} s_{2}, t_{3}\right) \in R_{r}\left(t_{1}, t_{3}\right)$ and the composition is associative where defined. The identity arrow of $R_{r}(t, t)$ is $(t, 1, t)$ where 1 is the identity of $S$. So, $R_{r}$ is a category. Let $\alpha$ be a congruence on $S$ and for $\left(t_{1}, s_{1}, t_{2}\right),\left(t_{1}, s_{2}, t_{2}\right) \in R_{r}\left(t_{1}, t_{2}\right)$ define $\left(t_{1}, s_{1}, t_{2}\right) \Omega\left(t_{1}, s_{2}, t_{2}\right)$ if and only if $s s_{1}=s s_{2}$ for all $s \in t_{1}^{r-1}$ and $s_{1} \alpha s_{2}$. Then, by [26, Lemma 25], $\Omega$ is a congruence on the category $R_{r}$. Let $D_{r}^{\alpha}=R_{r} / \Omega$. Following Tilson [18], we term $D_{r}^{\alpha}$ the derived category of $r$. Let $\left[t_{1}, s_{1}, t_{2}\right] \in D_{r}^{\propto}\left(t_{1}, t_{2}\right)$ denote the $\Omega$-class of $\mathbb{R}_{r}$ containing $\left(t_{1}, s_{1}, t_{2}\right) \in \mathbb{R}_{r}\left(t_{1}, t_{2}\right)$. We define $x \lambda y$ (in $\widehat{S}$ ) if $x, y \in F_{v}$ for soine $v$. Clearly, $\lambda$ is a congruence relation on $\widehat{S}$.

Lemma 1.10. For $t \in(\widehat{S / \delta}),[t, s, t] \tau=s L$ defines an isomorphism of $D_{\hat{\delta}}^{\lambda}(t, t)$ onto $\left(U_{t} \widehat{\delta}^{-1} / L\right)^{1}$.

Proof. Suppose $s L z\left(s, z \in U_{t} \widehat{\delta}^{-1}\right)$ Hence, using Lemma 1.8, $s, z \in W_{j}$ for some ${ }_{j} \in$ $\tilde{P}_{|t|}$. Thus, using modified [26, Lemma 23], $s \sigma^{\prime} z$. Hence, $x s=x z$ for all $x \in W_{\vec{k}}$

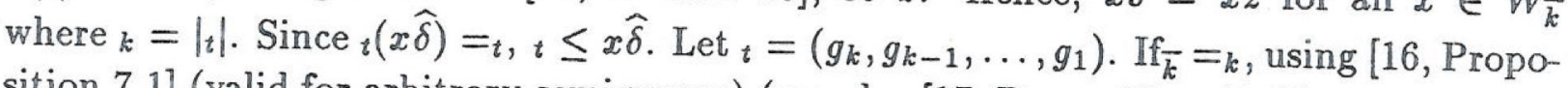
sition 7.1] (valid for arbitrary semigroups) (see also [17, Proposition 12.1]), Lemmas 1.61.8, and [24, Lemma 3.1[f], $x \widehat{\delta}=\left(e_{k}, g_{k-1}, \ldots, g_{1}\right)$ where $e_{k}^{2}=e_{k} L g_{k}$. Using Lemmas 1.5, 1.6 and 1.8 if $u \in_{t} \widehat{\delta}^{-1}$, then $u=\left(\left(g_{k} ; i_{k}, j_{k}\right),\left(g_{k-1} ; i_{k-1}, j_{k-1}\right), \ldots,\left(g_{1} ; i_{1}, j_{1}\right)\right)$, say. Since 
$W_{\bar{k}}=W_{k}=E\left(F_{k^{\prime}}\right) \cap U_{t} \widehat{\delta}^{-1}$ (where $k \longrightarrow k^{\prime}$ defines isomorphism of $\widetilde{P}_{k}$ into $Y$ ) (see [26]), let $x=\left(\left(e_{k} ; i_{k}, j_{k}\right),\left(g_{k-1} ; i_{k-1}, j_{k-1}\right), \ldots,\left(g_{1} ; i_{1}, j_{1}\right)\right)$. Since $\left(g_{k} ; i_{k}, j_{k}\right) L\left(e_{k} ; i_{k}, j_{k}\right)$, it is easily checked that $u x=u$. Hence, $u s=u x s=u x z=u z$. Since $s, z \in W_{j}, s \lambda z$. Thus, $\left[{ }_{t}, s, t_{t}\right]=\left[{ }_{t}, z, t\right]$. Next, assume ${ }_{k}>_{\bar{k}}$. Then, using [17, Proposition 12.1]), Lemma 1.7 and [24, Theorem 3.1(f)], $t=\left(g_{k}, g_{k-1}, \ldots, g_{\bar{k}}, g_{\bar{k}-1}, \ldots, g_{1}\right)$ and $x \widehat{\delta}=\left(e_{\bar{k}}, g_{\bar{k}-1}, \ldots, g_{1}\right)$ where $g_{\bar{k}} L e_{\bar{k}}=e \frac{2}{k}$. Herice, $u=\left(g_{k} ; i_{k}, j_{k}\right),\left(g_{k-1} ; i_{k-1}, j_{k-1}\right), \ldots,\left(g_{\bar{k}}, i_{\bar{k}}, j_{\bar{k}}\right),\left(g_{\bar{k}-1} ; i_{\bar{k}-1}, j_{\bar{k}-1}\right)$, $\left.\ldots,\left(g_{1} ; i_{1}, j_{1}\right)\right)$ and $x=\left(\left(e_{\bar{k}} ; i_{\bar{k}}, j_{\bar{k}}\right),\left(g_{\bar{k}-1} ; i_{\bar{k}-1}, j_{\bar{k}-1}\right), \ldots,\left(g_{1} ; i_{1}, j_{1}\right)\right)$.

Since $\left(g_{s} ; i_{s}, j_{s}\right)<\left(g_{\bar{k}} ; i_{\bar{k}}, j_{\bar{k}}\right)$ for $\bar{k}<_{s} \leq_{k},\left(g_{s} ; i_{s}, j_{s}\right)\left(e_{\bar{k}} ; i_{\bar{k}}, j_{\bar{k}}\right)=\left(g_{s} ; i_{s}, j_{s}\right)$. Furthermore $\left(g_{\bar{k}} ; i_{\bar{k}}, j_{\bar{k}}\right) L\left(e_{\bar{k}} ; i_{\bar{k}}, j_{\bar{k}}\right)$. Hence, by a routine calculation, $u x=u$. Thus, as above, $\left[{ }_{t}, s, t\right]=\left[{ }_{t}, z_{\lambda^{t}}\right]$. Conversely, assume $[t, s, t]=[t, z, t]$. Hence, $s, z \in F_{q}$, say and $x s=x z$ for all $x \in_{t} \widehat{\delta}^{-1}$. Using [26, Lemma 22], $s \leq z$ or $z \leq s$. Using Lemma 1.7, $s z=s$ or $z s=z$. Since $s, z \in W_{j}$ for some $j, s L z$ in either case. Thus, $[t, s, t] \tau=s L\left(s \in U_{t} \widehat{\delta}^{-1}\right)$ defines a 1-1 map of $D_{\hat{\delta}}^{\lambda}(t, t)$ into $\left(U_{t} \widehat{\delta}^{-1} / L\right)^{1}$. Clearly, $\tau$ is a surjection. Using Lemma $1.9, \tau$ is an isomorphism.

Theorem 1.11. Let $S$ be a super quasi-adequate semigroup. Then,

$$
S^{1}<V \circ(\widehat{S / \delta})^{1}
$$

where $V$ is an L-trivial and idempotent monoid, $\delta$ is the minimum adequate good congruence on $S,(\widehat{S / \delta})$ is a semilattice $Y=S / J^{*}$ of left cancellative semigroups $\left(X_{y}: y \in Y\right)$ with idempotents, and $E((\widehat{S / \delta}))$ is a semilattice $Y$ of right zero semigroups $\left(E\left(X_{y}\right):_{y} \in\right.$ Y).

Proof. Utilize Lemma 1.5 (define $1 \delta=1$ ), Lemma 1.10, [26, Lemma 29], and [26, Theorem 26] to establish (1). To complete the proof utilize Proposition 1.3 and Lemma 1.5 .

Remark 1.12. If $E$ is the edge set of the graph obtained from $D_{\hat{\delta}}^{\lambda}$ by removing the identity arrows, then $V$ is the free monoid over $E$ relative to the equation $x y x=$ $y x\left(x, y \in E^{1}\right)$ (see [26]-especially the proof of [26, Lemma 29]). $V$ is a semilattice $A$ (set of all finite subsets of $E$ under union) of right zero semigroups ( $U_{\rho}: P \in A$ ) where $U_{p}$ denotes the set of all elements of $V$ with content $P$ (see [2], [10] and [26, especially Theorem 27])

Lemma 1.12. $X_{y}=C_{y} \times E_{y}$ where $C_{y}$ is a cancellative monoid and $E_{y}$ is a right zero semigroup if and only if $T_{y}$ is a group. In the case, $X_{y}=T_{y} \times E\left(X_{y}\right)$.

Proof. Suppose $X_{y}=C_{y} \times E_{y}$. Then, Using [19, Theorem 2], $a \in a X_{y}$ for all $a \in X_{y}$. Thus, $\left(a_{n}\right)=\left(a_{n}\right) e$ for some $e \in X_{y}$. Hence, $\left(a_{n}\right) e=\left(a_{n}\right) e^{2}$. Thus, using Lemma 1.5, $e=e^{2}$. Hence, using Lemma 1.5, $\left(a_{n}\right)=\left(a_{n}\right)\left(e_{y}, x_{k-1}, \ldots, x_{1}\right)$ where $e_{y}$ is the identity of $T_{y}$. Thus, $\left(a_{n}\right)=\operatorname{red}\left(a_{n}, e_{y}, x_{k-1}, \ldots, x_{1}\right)$. So, $a_{n} L e_{y}$. Hence, using Lemma $1.4, e_{y}=s a_{n}$ where $s$ may be taken as an element of $T_{y}$. Thus, $a_{n} s a_{n} s=$ 
$a_{n} e_{y} s=a_{n} s=a_{n} s e_{y}$. So, $a_{n} s=e_{y}$ and, hence, $T_{y}$ is a group. Conversely, suppose $T_{y}$ is a group. Let $\left(a_{n}, a_{n-1}, \ldots, a_{1}\right) \in X_{y}$. Then, $\left(a_{n}, a_{n-1}, \ldots, a_{1}\right)=\left(a_{n}\right)\left(e_{y}, a_{n-1}, \ldots, a_{1}\right)$. Since $\left(a_{n}\right)\left(b_{n}\right)=\left(a_{n} \cdot b_{n}\right)$ for $a_{n}, b_{n} \in T_{y}, T_{y} \cong\left\{\left(a_{n}: a_{n} \in T_{y}\right\}\right.$. Thus, it is easily checked that every element of $X_{y}$ may be uniquely expressed in the form (a)e where $a \in T_{y}$ and $e \in E\left(X_{y}\right)$ and $(a, e) \longrightarrow(a) e$ defines an isomorphism of $T_{y} \times \mathbb{E}\left(X_{y}\right)$ onto $X_{y}$.

Remark 1.13. In the case $S$ is an orthodox union of groups in Theorem $1.11, \delta$ becomes the minimum inverse semigroup congruence on $S, J^{*}=J$ and $X_{y}=T_{y} \times \mathbb{E}\left(X_{y}\right)$ where $T_{y}$ is a maximal subgroup of $S$ (hence, $X_{y}$ is a right group). These facts are a consequence of Proposition 1.3. Lemma 1.1, and Lemma 1.12. In this case, the structure of $(\widehat{S / \delta})$ is further refined by [25, Theorem 2.6] (see also [26, Theorem 31]).

\section{Section 2. The Structure of Super Generalized $L^{*}$-unipotent Semigroups.}

In this section, we describe the smallest $L^{*}$-unipotent good congruence on a super generalized $L^{*}$-unipotent semigroup (Proposition 2.1) and give a structure theorem for super generalized $L^{*}$-unipotent semigroups (Theorem 2.4).

Proposition 2.1. Let $S$ be a super generalized $L^{*}$-unipotent semigroup. Then, $\delta \cap \mathbb{L}$ is the smallest $L^{*}$-unipotent good congruence on $S . S / \delta \cap L$ is a semilattice $Y=S / J^{*}$ of semigroups $\left(M_{y}: y \in Y\right)$ where $M_{y}=T_{y} \times J_{y}$ where $T_{y}$ is the cancellative monoid of Lemma 1.1 and $J_{y}$ is an $R$-class of $E\left(S_{y}\right) . E(S / \delta \cap L)$ is a semilattice $Y$ of the right zero semigroups $\left(J_{y}: y \in Y\right)$.

Proof. We first show that $\delta \cap L$ is a congruence relation on $S$. Utilizing Proposition 1.3, $\delta \cap L$ is a right congruence relation on $S$. Let $\overline{\delta \cap L}$ be the smallest congruence relation on $S$ containing $\delta \cap L$. We will show that $\overline{\delta \cap L}=\delta \cap L$. Suppose a $(\overline{\delta \cap L}) b$. Then, there exists $a=a_{1}, a_{2}, \ldots, a_{n}=b \in S$ such that $a_{i}=$ $x_{i} u_{i}, a_{i+1}=x_{i} v_{i}$ where $x_{i} \in S^{1}$ and $\left(u_{i}, v_{i}\right) \in \delta \cap L$ for $1 \leq i \leq n-1$. Let $x_{i}=(g ; i, k)_{\gamma} \in S_{\gamma}, u_{i}=(w ; s, j)_{\lambda} \in S_{\lambda}$, and $v_{i}=(w ; t, j)_{\lambda} \in S_{\lambda}$. Since $\delta$ is a. congruence relation, $a_{i}=(m ; p, q)_{\gamma \lambda}$ and $a_{i+1}=(m ; i, d)_{\gamma \lambda}$, say. Let $\alpha={ }_{\gamma \lambda}$. Then, $\alpha \gamma={ }_{\alpha \lambda}=\alpha$. Hence, $(m ; p, q)_{\alpha}=(g ; i, k)_{\gamma}\left(e_{\gamma} ; i, k\right)_{\gamma}\left(e_{\lambda} ; s, j\right)_{\lambda}(w ; s, j)_{\lambda}$ and $(m ; c, d)_{\alpha}=(g ; i, k)_{\gamma}\left(e_{\gamma} ; i, k\right)_{\gamma}\left(e_{\lambda} ; t, j\right)_{\lambda}(w ; s, j)_{\lambda}$ where $e_{\gamma}$ is the identity of $T_{\gamma}$.

Since $L$ is a congruence relation on $E(S),\left(e_{\gamma} ; i, k\right)_{\gamma}\left(e_{\lambda} ; s, j\right)_{\lambda} L\left(e_{\gamma} ; i, k\right)_{\gamma}\left(e_{\lambda} ; t, j\right)_{\lambda}$. Hence, $\left(e_{\lambda} ; i, k\right)_{\gamma}\left(e_{\lambda} ; s, j\right)_{\lambda}=\left(e_{\alpha} ; s^{\prime}, j^{\prime}\right)_{\alpha}$ and $\left(e_{\gamma} ; i, k\right)_{\gamma}\left(e_{\lambda} ; t, j\right)_{\lambda}=\left(e_{\alpha} ; t^{\prime}, j^{\prime}\right)_{\alpha}$, say. Hence,

$$
\begin{aligned}
& (m ; p, q)_{\alpha}=(g ; i, k)_{\gamma}\left(e_{\alpha} ; s^{\prime}, j^{\prime}\right)_{\alpha}(w ; s, j)_{\lambda} \\
& (m ; c, d)_{\alpha}=(g ; i, k)_{\gamma}\left(e_{\alpha} ; t^{\prime}, j^{\prime}\right)_{\alpha}(w ; s, j)_{\lambda}
\end{aligned}
$$

Since $L$ is a right congruence relation on $S,\left(e_{\alpha} ; s^{\prime}, j^{\prime}\right)_{\alpha}(w ; s, j)_{\lambda} L\left(e_{\alpha} ; t^{\prime}, j^{\prime}\right)_{\alpha}(w$; $s, j)_{\lambda}$. Hence, $\left(e_{\alpha} ; s^{\prime}, j^{\prime}\right)_{\alpha}(w ; s, j)_{\lambda}=\left(w^{*} ; s^{*}, j^{*}\right)_{\alpha}$ and $\left(e_{\alpha} ; t^{\prime}, j^{\prime}\right)_{\alpha}(w ; s, j)_{\lambda}=\left(\bar{w} ; \bar{s}, j^{*}\right)_{\alpha}$, 
say. Thus,

$$
\begin{aligned}
& (m ; p, q)_{\alpha}=(g ; i, k)_{\gamma}\left(w^{*} ; s^{*}, j^{*}\right)_{\alpha} \\
& (m ; c, d)_{\alpha}=(g ; i, k)_{\gamma}\left(w ; s, j^{*}\right)_{\alpha}
\end{aligned}
$$

Hence,

$$
\begin{aligned}
& \left(e_{\alpha} ; p, q\right)_{\alpha}(m ; p, q)_{\alpha}=\left(e_{\alpha} ; p, q\right)_{\alpha}(g ; i, k)_{\gamma}\left(w^{*} ; s^{*}, j^{*}\right)_{\alpha} \\
& \left(e_{\alpha} ; p, q\right)_{\alpha}(m ; c, d)_{\alpha}=\left(e_{\alpha} ; p, q\right)_{\alpha}(g ; i, k)_{\gamma}\left(\bar{w} ; \bar{s}, j^{*}\right)_{\alpha} .
\end{aligned}
$$

Suppose that $\left(e_{\alpha} ; p, q\right)_{\alpha}(g ; i, k)_{\gamma}=(\bar{g} ; \bar{i}, \bar{k})_{\alpha}$. Then,

$$
\begin{aligned}
& (m ; p, q)_{\alpha}=(\bar{g} ; \bar{i}, \bar{k})_{\alpha}\left(w^{*} ; s^{*}, j^{*}\right)_{\alpha} \\
& (m ; p, d)_{\alpha}=(\bar{g} ; \bar{i}, \bar{k})_{\alpha}\left(\bar{w} ; \bar{s}, j^{*}\right)_{\alpha}
\end{aligned}
$$

Hence, $q=d=j^{*}$. Thus, $a_{i}(\delta \cap L) a_{i+1}$ for $1 \leq i \leq n-1$. Hence, $a(\delta \cap L) b$ and, thus $\delta \cap L=\overline{\delta \cap \bar{L}}$.

We will need to show that $\delta \cap L^{*}=\delta \cap L$. Suppose $a\left(\delta \cap \mathbb{L}^{*}\right) b$. Since $a \delta b, a=$ $(g ; i, j)_{\alpha} \in S_{\alpha}$ and $b=(g ; r, s)_{\alpha} \in S_{\alpha}$, say. There exists an oversemigroup $S^{*}$ of $S$ such that $s(g ; i, j)_{\alpha}=(g ; r, s)_{\alpha}$ where $s \in S^{*}$. Hence, $(g ; r, s)_{\alpha}\left(e_{\alpha} ; i, j\right)_{\alpha}=(g ; r, s)_{\alpha}$.

Thus, $j=s$. Hence, $a(\delta \cap L) b$. Thus, $\delta \cap L^{*} \leq \delta \cap L$. Since $L \leq L^{*}, \delta \cap L^{*}=\delta \cap L$.

We next show that $\delta \cap L$ is a good congruence. We will use [5, Corollary 1.5].

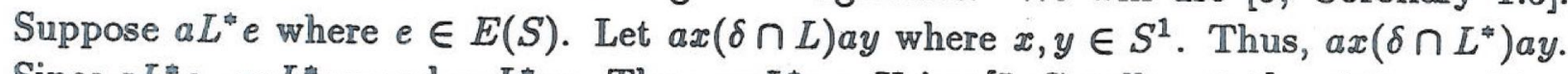
Since $a L^{*} e$, $a x L^{*} e x$ and $a y L^{*} e y$. Thus, ex $L^{*} e y$. Using [5, Corollary 1.5] and Proposition 1.3, ex $\delta$ ey for some $e^{2}=e \in L^{*} a$. Thus, ex $\left(\delta \cap L^{*}\right) e y$. Hence, ex $(\delta \cap L) e y$. Next, let $a R^{\text {t }} e$ where $e \in E(S)$. Assume $x a(\delta \cap L) y a$ where $x, y \in S^{1}$. Thus, $x a=(h ; m, n)_{\alpha}$ and $y a=(h ; p, n)_{\alpha}$, say. Let $f=\left(e_{y} ; m, n\right)_{\alpha}$. Then, $x a=f y a$. Hence, $f x a=f y a$. Thus, using [11, Lemma 1.7], $f x e=f y e$. Since $x a R^{*} x e$ and ya $\mathbb{R}^{*} y e$, it is easily seen that $x e, y e$, and $f \in S_{\alpha}$. Hence $f x e=f y e$ implies $x e(\delta \cap L) y e$. Thus, $\delta \cap L$ is a good congruence on $S$ by [5, Corollary 1.5].

We next show that $S / \delta \cap \mathbb{L}$ is an $L^{*}$-unipotent semigroup. Using [6, Proposition 1.6], $S / \delta \cap L$ is a quasi-adequate semigroup. Using [6, Lemma 1.5], $E(S / \delta \cap L)=\{e(\delta \cap L)$ : $e \in \mathbb{E}(S)\}$. Suppose $e(\delta \cap L) L f(\delta \cap L)$ (in $E(S / \delta \cap L)$ ). Thus, $(e f, e) \in \delta \cap L$ and $(f e, f) \in \delta \cap \mathbb{L}$. Hence, $e, f \in S_{\gamma}$, say. Thus, $e=e f e=e f$. Hence, $e(\delta \cap L) f$. Thus, $S / \delta \cap \mathbb{L}$ is an $\mathbb{L}^{*}$-umipotent semigroup.

Let $e$ be an $L^{*}$-unipotent congruence on $S$. Suppose $a(\delta \cap L) b$. Then, $a=(g ; m, n)_{\alpha}$ and $b=(g ; p, n)_{\alpha}$, say. Thus $a=\left(e_{\alpha} ; m, n\right)_{\alpha} b$. Since $\left(e_{\alpha} ; m, n\right)_{\alpha} L\left(e_{\alpha} ; p, n\right)_{\alpha},\left(e_{\alpha} ; m, n\right)_{\alpha} e$ $=\left(e_{\alpha} ; p, n\right)_{\alpha} e$. Hence, $a e=\left(e_{\alpha} ; m, n\right)_{\alpha} e b e=\left(e_{\alpha} ; p, n\right)_{\alpha} b e=b e$. Thus, $\delta \cap L \leq e$. Thus, $\delta \cap L$ is the smallest $L^{*}$-unipotent congruence on $S$.

Using Lemma 1.1, $S_{y}=T_{y} \times I_{y} \times J_{y}$ (algebraic direct product) where $I_{y}$ is a left zero semigroup and $J_{y}$ is a right zero semigroup. Let $M_{y}=T_{y} \times J_{y}$ (algebraic direct product). Let $(\overline{g ; i, j})$ denote the $\delta \cap L$-class of $S$ containing $(g ; i, j)$. Then, $(\overline{g ; i, j}) \lambda=(g, j)$ defines a 1-1 mapping of $S / \delta \cap L$ onto $M=U\left(M_{y}: y \in Y\right)$. In a similar manner to the proof of Lemma 1.4, we may. define a multiplication on $M$ such that $M$ is a semilattice $Y$ of the 
semigroups $\left(M_{y}: y \in Y\right)$ and $M \cong S / \delta \cap L$. The last sentence follows since $E(M)$ is a
semigroup.

Remark 2.2 will be used in the proof of Theorem 2.4.

Remark 2.2. Let $\theta$ be a homomorphism of a semigroup $S$ onto a semigroup $T$. Define $D(\theta)=\left\{(t, s, t(s \theta)): t \in \mathbb{T}^{\circ} ; s \in S\right\} U\{O\}$ under the multiplication $\left(t_{1}, s_{1}\right.$, $\left.t_{1}\left(s_{1} \theta\right)\right)\left(t_{2}, s_{2}, t_{2}\left(s_{2} \theta\right)\right)=\left(t_{1}, s_{1} s_{2}, t_{1}\left(s_{1} s_{2}\right) \theta\right)$ if $t_{1}\left(s_{1} \theta\right)=t_{2} ; 0$ if $t_{1}\left(s_{1} \theta\right) \neq t_{2}$ and $O(t, s, t(s \theta))=(t, s, t(s \theta)) O=0 . O=O . D(\theta)$ was termed the derived semigroup of $\theta$ by its inventor Bret Tilson (see [16] and [17]). Let $\phi$ be a mapping of $D(\theta)-\{0\}$ into a semigroup P. Following Rhodes [13, Definition A.I.2.1, p. 94], we term $\phi: D(\theta)-\{O\} \rightarrow \mathbb{P}$ a parametrization of $D(\theta)$ if 1$) \phi$ is a partial homomorphism of $D(\theta)-\{0\}$ into $\mathbb{P}$ (i.e. if $x, y \in D(\theta)-\{O\}$ and $x y \neq 0$, then $x \theta y \theta=(x y) \theta)$ 2) $\phi$ satisfies the embedding condition: $s_{1} \theta=s_{2} \theta$ and $\left(t, s_{1}, t\left(s_{1} \theta\right)\right) \phi=\left(t, s_{2}, t\left(s_{2} \theta\right)\right) \phi$ for all $t \in T^{*}$ implies $s_{1}=s_{2}$. For brevity, we also term $P$ a parametrization of $D(\theta)$. Using [13, Proposition AI.2.3], $S \leq P$ PT where $p \mid S=\theta$ ( $p$ is the projection if $P o T$ onto $T)$. Following Rhodes [13], we define $D^{\mathbb{R}}(\theta)$ (dual derived semigroup) as follows: $D^{R}(\theta)=\left(((s \theta) t, s, t): s \in S, t \in \mathbb{T}^{\bullet}\right) U\{0\}$ under the multiplication $\left(\left(s_{1} \theta\right) t_{1}, s_{1}, t_{1}\right)\left(\left(s_{2} \theta\right) t_{2}, s_{2}, t_{2}\right)=\left(\left(s_{1} \theta\right) t_{1}, s_{1} s_{2}, t_{2}\right)$ if $t_{1}=\left(s_{2} \theta\right) t_{2}$; 0 if $t_{1} \neq\left(s_{2} \theta\right) t_{2} ; o((s \theta) t, s, t)=((s \theta) t, s, t) o=00=0$. A parametrization $P^{R}$ of $D^{R}(\theta)$ is defined as above and $S \leq T \circ P^{R}$ with $p \mid S=\theta$.

Remark 2.3 will be needed for the statement of Theorem 2.4

Remark 2.3. Let $W$ be a partial groupoid which is a union of a collection of pairwise disjoint subsemigroups $\left(T_{y}: y \in Y\right)$ where $Y$ is a semilattice. If $a \in T_{y}, b \in T_{x}$ and $y \geq x$ (in $Y$ ) imply $a b$ is defined (in $W$ ) and $a b \in T_{x}$ and $x \geq w$ and $c \in T_{w}$ imply $(a b) c=a(b c)$, we term $W$ a lower partial chain $Y$ of the semigroups $\left(T_{y}: y \in Y\right)$. Let $X$ be a semilattice $Y$ of semigroups $\left(X_{y}: y \in Y\right)$ and let $R$ and $S$ be semigroups. For the definition of $W \circ X \circ R$ and $S \leq W \circ X \circ R$, see [24, p. 188 and p. 189].

Theorem 2.4. Let $S$ be a super generalized $L^{*}$-unipotent semigroup. Then, (1) $S \leq W^{1} o(E(S) / L)^{1} o(S / \delta \cap L)^{1}$ where $W$ is a lower partial chain $Y=S / J^{\text {t }}$ of left zero subsemigroups of $E(S), E(S) / L$ is a semilattice $Y$ of right zero semigroups, and $\delta \cap \mathbb{L}$ is the smallest $L^{*}$-unipotent good congruence on $S$. Furthermore, (2) $S / \delta \cap L \leq(S / \delta \cap L / e)^{1} o(E(S) / L)^{1}$ where $e$ is the smallest adequate good congruence on $S / \delta \cap L$ and $S / \delta \cap L / e$ is a strong semilattice $Y$ of cancellative monoids $\left(T_{y}: y \in Y\right)\left(T_{y}\right.$ is a cancellative subsemigroup of $S$ ).

Proof. We will first establish that $S \leq(E(S))^{1} o(S / \delta \cap L)^{1}$. For each $(g, j)_{y} \in$ $M_{y}\left({ }_{y} \in Y\right)$ (Notation of Proposition 2.1), select a representative element $u_{(g, j) y}$ in $S_{y}$. We
first show that every element of $S$ may be uniquely ex first show that every element of $S$ may be uniquely expressed in the form $w_{(e y, j) y} w_{(e y, j) y} \in\left(e_{y}, j\right)_{y}(\delta \cap L)^{-1}$. where $w_{(e y, j) y} \in\left(e_{y}, j\right)_{y}(\delta \cap L)^{-1}$. Let $(g ; i, j)_{y} \in S_{y}$ and suppose $u_{(g, j) y}=\left(g ; i_{o}, j\right)_{y}$. Then, $(g ; i, j)_{y}=\left(e_{y} ; i, j\right)_{y}\left(g ; i_{o}, j\right)_{y}$ where $\left(e_{y} ; i, j\right)_{y} \in\left(e_{y}, j\right)_{y}(\delta \cap L)^{-1}$. It is easily checked that the above expression is unique. If $s=(g, j)_{y}$, let ${ }_{s}^{+}=\left(e_{y}, j\right)_{y}$. Thus every element of $S$ may be uniquely expressed in the form $w_{s}^{+} u_{s}$ where $w_{s}^{+} \epsilon_{s}^{+}(\delta \cap L)^{-1}$. 
Let $t \in S / \delta \cap L$ and $s \epsilon_{x}(\delta \cap L)^{-1}$. Hence, we may write $u_{t}^{s}=f(t, s) u_{t x}$ where $\left.f(t, s) \in{ }_{t}\right)^{+}(\delta \cap L)^{-1}$. First assume $S$ has an identity. For $\left({ }_{t, s, t}(s(\delta \cap L))\right) \in D(\delta \cap$ $L)-\{O\}$, define $\left({ }_{t, s, t}(s(\delta \cap L))\right) \theta=f\left({ }_{t, s}\right)$. We will show that $\theta: D(\delta \cap L)-\{O\} \rightarrow$ $E(S)$ is a parametrization of $D(\delta \cap L)$. It is easily checked that $\theta$ defines a mapping of $D(\delta \cap L)-\{O\}$ into $E(S)$. Next, we show that $\theta$ defines a partial homomorphism. Let $\left.\left(t_{1}, s_{1}, t_{1}\left(s_{1}(\delta \cap L)\right)\right) ;\left(t_{2}, s_{2}, t_{2}\left(s_{2} \delta \cap L\right)\right)\right) \in D(\delta \cap L)$ with $t_{t_{1}}\left(s_{1}(\delta \cap L)\right)=t_{t_{2}}$. We must show $f\left(t_{1}, s_{1}\right) f\left(t_{2}, s_{2}\right)=f\left(t_{1}, s_{1}, s_{2}\right)$. Suppose $s_{1} \in_{x_{1}}(\delta \cap L)^{-1}$ and $s_{2} \in_{x_{2}}(\delta \cap L)^{-1}$. Then, $u_{t_{1}}\left(s_{1}, s_{2}\right)=f\left(t_{1}, s_{1} s_{2}\right) u_{t_{1} x_{1} x_{2}}=f\left(t_{1}, s_{1} s_{2}\right) u_{t_{2} x_{2}}$ where $f\left(t_{1}, s_{1} s_{2}\right) \in\left(t_{2} x_{2}\right)^{+}(\delta \cap L)^{-1}$. However, $\left(u_{t_{1}} s_{1}\right) s_{2}=f\left(t_{1}, s_{1}\right)\left(u_{t_{2}} s_{2}\right)=f\left(t_{1} s_{1}\right) f\left(t_{2}, s_{2}\right) u_{t_{2} x_{2}}$. Let $t_{2} \in M_{y}$ and $x_{2} \in M_{x}$, say. Hence, $t_{t_{2} s_{2}} \in M_{y x}$. Furthermore, $t_{t_{2}}^{t} \in E\left(M_{y}\right)$ and $\left(t_{2} s_{2}\right)^{+} \in E\left(M_{y x}\right)$. Using the last sentence of Proposition 2.1, ${ }_{t_{2}}^{+}\left(t_{2} x_{2}\right)^{+}=\left({ }_{t_{2}}^{+}\left(t_{2} x_{2}\right)^{+}\right)\left(t_{t_{2} x_{2}}\right)^{+}=\left(t_{2} x_{2}\right)^{+}$. Hence, $f\left(t_{1}, s_{1}\right) f\left(t_{2}, s_{2}\right) \in\left(t_{2} t_{2}\right)+(\delta \cap L)^{-1}$. Thus, $f\left(t_{1}, s_{1}\right) f\left(t_{2}, s_{2}\right)=f\left(t_{1}, s_{1} s_{2}\right)$, and, hence, $\theta$ is a partial homomorphism. We next show the embedding condition is valid. Let $e$ denote the identity of $S / \delta \cap L$ and let $u_{e}=1$, the identity of $S$. Thus, if $s_{1}(\delta \cap L)=_{s_{2}}(\delta \cap L)=x_{x}$ and $f\left(e, s_{1}\right)=f\left(e, s_{2}\right)$, then $s_{1}=u_{e} s_{1}=f\left(e, s_{1}\right) u_{x}=f\left(e, s_{2}\right) u_{x}=u_{e} s_{2}=s_{2}$. Hence, $E(S)$ is a parametrization of $D(\delta \cap L)$. Thus, using Remark 2.2, $S \leq E(S) \circ S / \delta \cap L$. If $S$ has no identity consider $S^{1}$. Note that $a(\delta \cap L)_{1}\left(\right.$ in $\left.S^{1}\right)$ implies $a=1$. Hence, $S^{1} / \delta \cap L \cong$ $(S / \delta \cap L)^{1}$. Furthermore, $E\left(S^{1}\right) \cong(E(S))^{1}$. Hence, $S \leq S^{1} \leq(E(S))^{1} \circ(S / \delta \cap L)^{1}$. Thus utilizing [24, Theorem 1.24, Remark $(1.24)^{\prime}$, Lemma 1.23, and Lemma 1.25], we obtain (1). We next establish (2). Let $M=S / \delta \cap L$. Utilizing [9, Corollary 6.2 and Proposition 6.5], Proposition 2.1 and Lemma $1.4, M / e$ is the strong semilatice $Y$ of cancellative monoids $\left(T_{y}: y \in Y\right)$. If $s \in T_{y}$, let ${ }_{s}^{*}=e_{y}$, the identity of $T_{y}$. For each $s \in M / e$, select a representative element $u_{s} \in_{s} e^{-1}$. We show that every element of $M$ may be uniquely expressed in the form $u_{s} w_{s}^{*}$ where $w_{s}^{*} \in_{s}^{*} e^{-1}$. Let $(g, j)_{y} \in M_{y}$ and suppose $u_{s}=\left(g, j_{0}\right)_{y} \in M_{y}$. Hence, $(g, j)_{y}=\left(g, j_{0}\right)_{y}\left(e_{y}, j\right)_{y}$ where $\left(e_{y}, j\right)_{y} \in e_{y} e^{-1}$ and $g^{*}=e_{y}$. Suppose $u_{s} g_{s}^{*}=u_{s} h_{s}^{*}$. Then, since $M_{y}(y \in Y)$ is left cancellative, $g_{s}^{*}=h_{s}^{*}$. Let ${ }_{t} \in M / e$ and $s \epsilon_{x} e^{-1}$. Hence, we may write $u_{s} u_{t}=u_{x t} f(s, t)$ where $f(s, t) \in\left({ }_{x t}\right)^{*} e^{-1}$. First, assume that $M$ has an identity. For $\left(\left({ }_{s} e\right)_{t, s, t}\right) \in D^{R}(e)-\{O\}$, define $\left(\left({ }_{s} e\right)_{t, s, t}\right) \theta=f(s, t)$. Using the fact that $M / e$ is a strong semilattice $Y$ of cancellative monoids $\left(T_{y}:{ }_{y} \in Y\right)$, we proceed as above to show that $\theta: D^{R}(e)-\{O\} \rightarrow E(M)$ is a parametrization of $D^{R}(e)$. Thus, using Remark 2.2, $M \leq M / e^{\Omega} E(M)$. Again, proceeding as above, $M \leq M^{1} \leq(M / e)^{1{ }^{\Omega}}(E(M))^{1}$. Using Proposition 2.1, $E(M) \cong E(S) / L$. Hence (2) is valid. To complete the proof, utilize Proposition 2.1 .

Remark 2.5. W is a lower partial chain $Y$ of $L$-classes of $E(S)$. Each $J$-class of $E(S)$ contains precisely one of these $L$-classes (see [24, Theorem 1.24]).

Remark 2.6. Let $S$ be a generalized $L$-unipotent union of groups. Then, $\delta \cap L$ is the smallest $L$-unipotent congruence on $S$ ( $\delta$ is the smallest inverse semigroup congruence on $S)$, e is the smallest inverse semigroup congruence on $S / \delta \cap L, T_{y}$ is a maximal subgroup of $S$, and $J^{*}=J$ in the statement of Theorem 2.4. Thus, Theorem 2.4 generalizes [24, Theorem 1.27, Theorem 1.28, and Theorem 1.26] in the case $S$ is also a union of groups 
(our structure theorem for generalized $L$-unipotent unions of groups). A different type structure theorem for generalized $R$-unipotent unions of groups is given in [22, Theorem 4.7].

\section{Section 3 Super $\mathbb{R}^{*}$-umipotent Semigroups}

In this section, we give a structure theorem for super $R^{*}$-unipotent semigroups (Theorem 3.1)

Theorem 3.1. Let $S$ be a super $R^{*}$-unipotent semigroup. Thus, $* S \leq(E(S))^{1} 0$ $(S / \delta)^{1}$ where $E(S)$ is a semilattice $Y=S / J^{\text {t }}$ of left zero semigroups, $\delta$ is the smallest adequate good congruence on $S$, and $S / \delta$ is a strong semilattice $Y$ of cancellative monoids $\left(T_{y}:{ }_{y} \in Y\right)\left(T_{y}\right.$ is a subsemigroup of $\left.S\right)$.

Proof. Using Lemma 1.1, $S_{y}=T_{y} \times E\left(S_{y}\right)$ where $E\left(S_{y}\right)$ is a left zero semigroup. Hence, by a routine calculation, $\delta \cap L=\delta$. Thus, utilizing the proof of Theorem 2.4, $*$ is valid. Use Proposition 1.3 and Lemma 1.4 to complete the proof.

Remark 3.2. Let $S$ be an $R$-unipotent union of groups. Then, $\delta$ is smallest inverse semigroup congruence on $S, T_{y}$ is a maximal subgroup of $S$, and $J=J^{*}$ in the statement of Theorem 3.1. Hence, Theorem 3.1 generalizes [24, Remark 1.14, Theorem 1.12, and Theorem 1.8] (our structure theorem for $R$-unipotent unions of groups). A different type structure theorem for $L$-unipotent unions of groups is given in [22, Theorem 7.2].

\section{References}

[1] G. L. Bailes, "Right Inverse Semigroups", J. Algebra 26, 492-507, 1963.

[2] A. H. Clifford and G. B. Prestion, "The Algebraic Theory of Semigroups", Math. Swrveys Amer. Math Sac. 7, vol I (AMS, Providence, RI, 1961).

[3] A. H. Clifford, "A Structure Theorem for Orthogroups", J. Pure Appl. Algebra 8, 23-50, 1976.

[4] S. Eilenberg, "Automato, Languages, and Machines", Vol B (Academic Press, New York, 1976)

[5] A. El-Qallali and J. B. Fountain, "Idempotent-Connected Abundant Semigroups", Proc. Roy. Soc. Edinburgh, Series A, 91, 79-90, 1981.

[6] A. El-Qallali and J. B. Fountain, "Quasi-Adequate Semigroups", Proc. Roy. Soc. Edinburgh, Series A, 91, 91-99, 1981.

[7] A. El-Qallali, "L" Unipotent Semigroups", J. Pure Appl. Algebra. 62(1989), 9-33.

[8] J. B. Fountain, "Adequate Semigroups", Proc. Edinburgh Math. Soc. 22, 113-125, 1979.

[9] J. B. Fountain, "Abundant Semigroups", Proc. London Math. Soc. (3), 44, 103-129, 1982.

[10] J. M. Howie, "An Introduction to Semigroup Theory" (Academic Press, London, 1976).

[11] D. B. McAlister, "One-to-one Partial Right Translations of a Right Cancellative Semigroup", J. Algebra 43, 231-251, 1976.

[12] F. Pastijn, "A Representation of a Semigroup by a Semigroup of Matrices Over a Group With Zero", Semigroup Forum, 10, 238-249, 1975.

[13] J. Rhodes, "Infinite Iteration of Matrix Semigroups II. Structure Theorem for Arbitrary Semigroups Up to Aperiodic Morphism", J. Algebra 100, 25-137, 1986. 
[14] M. B. Szendrei, "On Takizawa's Construction for E-Unitary $R$-unipotent Semigroups", Beitr, Algebre Geom. 20, 197-201, 1985.

[15] M. B. Szendrei, "E-Unitary R-unipotent Semigroups", Semigroup Forum 32, 87-96, 1985.

[16] B. Tilson, "On the Complexity of Finite Semigroups", J. Pure Appl. Algebra 5, 187-208, 1974.

[17] B. Tilson, "Complexity of Semigroups and Morphisms", Chapter 12 of 4, 1976.

[18] B. Tilson, "Categories as Algebra: An Essential Ingredient in the Theory of Monoips", J. Pure Appl Algebra, 48(1987), 83-198.

[19] R. J. Warne, "The Direct Product of Right Zero Semigroups and Certain groupoids", Amer. Math. Monthly 74, 160-164, 1967.

[20] R. J. Warne, "L-unipotent Semigroups", Nigerian J. Science 5, 245-248, 1972.

[21] R. J. Warne, "Generalized L-unipotent Semigroups", Bull. Della Unione Math. Ital. 5, 43-47, 1972.

[22] R. J. Warne, "On the Structure of Semigroups Which are Unions of Groups", Trans. Amer. Math. Soc. 186, 385-401, 1973; announced in Sernigroup Forum 5, 323-330, 1973.

[23] R. J. Warne, "Generalized L-Unipotent Bisimple Semigroups", Pacific J. Math 51, 631-648, 1974.

[24] R. J. Warne, "Embedding of Regular Semigroups in Wreath Products", J. Pure Appl. Algebra 29, $177-20 \%, 1983$.

[25] R. J. Warme, "Embedding of Regular Semigroups in Wreath Products, III", J. Pure Appl. Algebra 40, 301-320, 1986.

[26] R. J. Warne, "On the Structure of Orthodox Umions of Groups", arabian J. Sci and Engineering, In press (Technicalreport No. 117, Dept of mathematicl Scicnces University of Petroleom and minerals, Dhahran Saudi Arabia, March, 1990)

Department of Mathematical Sciences King Fahd University of Petroleum and Minerals Dhahran 31261, Saudi Arabia. 